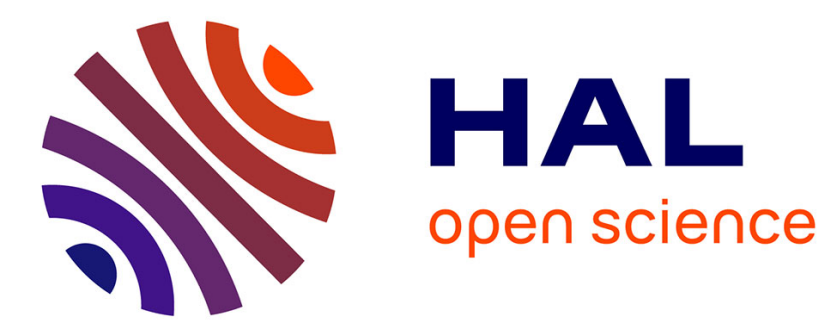

\title{
Prestations sociales, sécurité économique et croissance en Europe
}

Georges Menahem

\section{To cite this version:}

Georges Menahem. Prestations sociales, sécurité économique et croissance en Europe. Revue de l'OFCE, 2007, 103, pp.1-23. halshs-00198406

\section{HAL Id: halshs-00198406 https://shs.hal.science/halshs-00198406}

Submitted on 17 Dec 2007

HAL is a multi-disciplinary open access archive for the deposit and dissemination of scientific research documents, whether they are published or not. The documents may come from teaching and research institutions in France or abroad, or from public or private research centers.
L'archive ouverte pluridisciplinaire HAL, est destinée au dépôt et à la diffusion de documents scientifiques de niveau recherche, publiés ou non, émanant des établissements d'enseignement et de recherche français ou étrangers, des laboratoires publics ou privés. 


\section{Prestations sociales, sécurité économique et croissance en Europe}

Par Georges Menahem*

(Deuxième version pour publication $\mathrm{n}^{\circ} 103$ Revue de l'OFCE)

\section{Résumé}

Cet article évalue en quoi les prestations sociales contribuent à assurer une part importante de la sécurité économique des populations, notamment contre le risque de ne pas disposer de revenus suffisants. Il introduit pour cela un outil d'agrégation des garanties apportées par l'État social à la population, le «taux de sécurité démarchandisée». L'évaluation dans 20 pays européens de la diversité des ressources et des sécurités, dites démarchandisées parce qu'elles sont largement indépendantes du marché, permet de distinguer quatre grands types de contribution des prestations sociales à la sécurité économique. Les cinq pays du Nord assurent le maximum de garanties à leurs ressortissants, alors que les quatre pays du Sud et, plus encore, les cinq pays d'Europe continentale et orientale n'ont construit que des sécurités limitées, lesquelles sont associées à des risques de pauvreté plus importants. Les six pays dits « intermédiaires » ont connu des histoires hétérogènes qui leur permettent d'assurer un niveau important de sécurité économique à leurs populations. Des corrélations positives sont observées dans ces 20 pays européens entre, d'un coté, prestations sociales et sécurités indépendantes du marché et, d'un autre coté, meilleurs niveaux de productivité du travail et de niveaux de vie, ce qui contribue à rendre compte de la diversité de leurs taux de croissance.

\section{Introduction}

Selon tout un courant de pensée, les prestations sociales et les dépenses associées seraient excessives, en France et dans de nombreux autres pays européens. Au niveau théorique, l'existence d'un supposé «optimum de taxation» ${ }^{1}$ définirait un niveau de pression fiscale limite au-delà de laquelle le rendement de l'impôt diminuerait. L'effet dissuasif sur l'offre de travail l'emporterait alors sur l'effet de la hausse du niveau de taxation. En pratique, plus les charges sociales augmenteraient, plus les prix de revient des entreprises s'alourdiraient, ce qui diminuerait leur compétitivité, limiterait leur capacité à vendre leurs productions et donc réduirait les possibilités de croissance. Ces idées sont très influentes au niveau politique. Ce point de vue s'exprime en particulier au plus haut niveau de l'Union Européenne. Nous pouvons lire par exemple dans un rapport concernant l'avenir de la protection sociale en Europe : «Dans la plupart des pays européens, la sécurité sociale est essentiellement financée par les cotisations payées par les employeurs et les travailleurs. Et ces cotisations ont dépassé les 30\% du salaire brut dans la majorité des États ; dans certains pays tels que l'Allemagne et la France, elles sont même supérieures à $40 \%$. Un niveau aussi élevé de cotisations sociales a un impact négatif sur la croissance et la création d'emplois et favorise la persistance du chômage. Il gonfle le prix du travail pour les entreprises, freinant ainsi la demande de travail. $^{2} »$

Un autre courant de pensée adopte un point de vue allant au-delà du souci de l'équilibre des comptes des entreprises et de l'État: privilégiant une approche macroéconomique d'inspiration keynésienne, il est conduit à prendre davantage en compte la préoccupation du bien-être des populations et de la demande qui en résulte. Selon cette approche, les prestations sociales apparaissent plus comme un élément structurel des ressources des individus et des

\footnotetext{
* Directeur de recherche au CNRS - CEPN -Université Paris 13 et chercheur à l'IRDES.

${ }^{1}$ Qui serait fondée sur la courbe de Laffer, cf. par exemple Saez (2004).

${ }^{2}$ Cf. Commission Européenne (2004, p. 60).
} 
ménages, lequel découlerait d'un «compromis institutionnalisé» entre les acteurs sociaux (André, 1995). Par là, les institutions de la Sécurité sociale pourraient être considérées comme un stimulant important de l'activité économique du fait qu'elles contribuent directement à l'alimentation de la demande finale à l'économie (Delorme ; André, 1983). De fait, dans les années 1950-1980 et un peu moins depuis, elles ont joué un rôle de stabilisation essentiel dans les périodes de ralentissement conjoncturel (Boyer, 2001). La hausse des dépenses sociales associées a contribué ainsi pour une part déterminante à la sécurité économique des individus en les protégeant contre le risque de ne pas disposer de revenus suffisants. De plus, selon Cornilleau (2006), en France «cela n'a pas eu de conséquences négatives pour la croissance et l'emploi du fait des réformes successives du financement qui ont conduit à un allègement de la charge pesant sur les bas salaires ». Ce faisant, les diverses prestations sociales apportent des éléments essentiels à la cohésion sociale en permettant à chacun de se sentir aidé et secouru contre les conséquences aléatoires des accidents de la vie quotidienne. Et nous savons que la cohésion d'un peuple est un des facteurs qui contribuent à la qualité de son travail, donc à sa productivité et, en définitive, à la croissance.

Comment choisir entre ces deux types différents d'interprétation des contributions des prestations sociales à la santé de l'économie ? Pouvons-nous être aidés en cela par l'observation comparative des divers exemples d'utilisation en Europe de ces prestations, que certains appellent les «charges sociales» quand d'autres les caractérisent comme des éléments «socialisés » du salaire ? Sans prétendre répondre complètement à ces questions, nous allons chercher dans cet article à y apporter de nouveaux éléments de réponse. À cette fin, nous allons faire un détour qui, espérons-le sera productif. Nous allons dans un premier temps chercher à analyser en quoi les garanties et ressources apportées par l'État social contribuent à la sécurité économique des ressortissants de son territoire et, en particulier, en quoi elles les protègent contre le risque de ne pas disposer de revenus suffisants. Ceci nous conduira à définir, à la suite de Esping-Andersen (1990), des ressources « démarchandisées », à savoir indépendantes des aléas du marché et des choix des entreprises, puis à construire un «taux de sécurité démarchandisée » afin de synthétiser protection contre la pauvreté et sécurité économique. Dans un deuxième temps, nous chercherons à analyser les variations de ce taux dans vingt pays européens. L'observation de la diversité des liens entre prestations sociales et sécurité économique débouchera sur la caractérisation de quatre groupes de pays inégaux à la fois selon le niveau de leurs prestations et celui des sécurités qu'ils procurent. Enfin, nous retrouverons notre question de départ : le constat de la diversité des relations entre sécurité et niveaux de productivité, d'un coté, et entre sécurité et niveaux de vie, d'un autre coté, nous permettra de proposer quelques conclusions concernant les contributions des prestations sociales à la production, à sa croissance et à la société plus généralement.

\section{Une évaluation de l'agrégation des garanties de l'État social : le taux de sécurité démarchandisée}

Risquer de tomber dans la pauvreté constitue une des premières insécurités des populations. Elle résulte en grande partie de l'instabilité et du caractère variable de leurs revenus et de leurs autres ressources économiques, ce qui aboutit fréquemment à la mise en cause de leurs capacités à disposer régulièrement des moyens pour se nourrir, se loger, se vêtir et subvenir à leurs besoins fondamentaux. L'histoire du développement de l'État social et de la mise en place successive de ses missions peut ainsi être lue à la lumière des avatars de la sécurité matérielle des citoyens, ou encore de leur insécurité (Castel, 2003). En particulier, il a été essentiel de construire des systèmes de protection sociale garanties par la puissance publique 
pour assurer aux populations la disposition d'un minimum de revenus dans les cas où elles étaient incapables de travailler du fait d'accidents, de maladies, de handicaps ou encore du trop grand âge. Ces ressources sont d'autant plus précieuses que leur disposition est inconditionnelle et n'est pas soumise aux aléas des commandes ou de la conjoncture des marchés. Dans ce sens, elles sont «démarchandisées », pour reprendre le concept de Polanyi (1944) tel qu'il a été développé et adapté aux caractères des États sociaux modernes à travers les travaux de Esping-Andersen (1990).

Pour développer son concept, ce sociologue suédois se fonde sur une analyse sociale très proche de l'analyse marxiste: "Quand la force de travail devient une marchandise, la possibilité pour les individus de survivre hors du marché est mise en jeu. Ceci constitue le problème essentiel et conflictuel de la politique sociale. » [...] «Le bien-être et la survie en viennent à dépendre de la bonne volonté d'une personne à engager une force de travail $»^{3}$. Ces fondements le conduisent à proposer une définition presque fonctionnaliste: «La démarchandisation survient lorsqu'un service est obtenu comme un dû et lorsqu'une personne peut conserver ses moyens d'existence sans dépendre du marché ${ }^{4}$. L'essentiel ici est de souligner en quoi ce concept montre clairement que la sécurité échappe aux aléas du marché et des décisions patronales quand elle relève du contrôle de l'État et devient « démarchandisée ».

Comment évaluer alors les résultats des politiques des États sociaux en ce qui concerne la protection qu'ils assurent à leurs ressortissants contre les problèmes découlant des aléas de l'existence? Car les outils synthétiques que fournissent les comptes nationaux sont insuffisants pour évaluer aussi bien les insécurités économiques que les sécurités. Divers indicateurs statistiques ont été proposés depuis les années 1980 pour suppléer aux insuffisances du PNB par habitant. Parmi eux, deux manifestent le souci de prendre en compte la sécurité des personnes.

D'abord, il faut citer le score de démarchandisation utilisé par Esping-Andersen (1990) dans son livre pour illustrer le bien-fondé de sa typologie des systèmes de protection sociale. Selon la page explicitant les conventions de sa construction, ce score peut difficilement remplir d'autres objectifs car il agrège des variables de statuts très divers, à la fois qualitatives et quantitatives, et qui concernent trois domaines très différents ${ }^{5}$. De plus, les conventions d'évaluation des trois scores dont il fait la moyenne n'étant pas indiquées dans le livre, il est impossible de les reproduire pour les tester sur d'autres dates et d'autres pays et, a fortiori, pour étudier les corrélations de la sécurité ressentie des personnes avec leur productivité.

Une autre tentative exemplaire résulte des travaux de deux chercheurs canadiens qui ont élaboré un indicateur de bien-être économique dont un des objectifs explicites est de mettre en évidence les inconvénients du PNB par habitant en tant que mesure du bien-être économique (Osberg ; Sharpe, 2002). Son principe consiste à faire la moyenne de quatre indicateurs synthétiques, portant respectivement sur les flux de consommation au sens large, les stocks de richesses (économique, humaine et environnementale), les inégalités et la pauvreté économiques, et l'insécurité économique (risques économiques liés au chômage, à la maladie, à la vieillesse, et à ceux des familles monoparentales). Pour évaluer l'insécurité, ces

\footnotetext{
${ }^{3}$ Esping-Andersen (1990), pages 51 et 52.

${ }^{4}$ Ibidem, p.35

${ }^{5}$ Les unes sont juridiques (les règles d'éligibilité à la capacité à avoir droit à des ressources), les autres institutionnelles (les conditions de durée de cotisation, la durée des droits garantis et la période d'attente nécessaire pour obtenir un tel revenu) et les dernières économiques (le niveau des revenus de remplacement et la part individuelle du financement des prestations) (opus cité, trad. fr., 1999, pp. 73-74. Il faut préciser que la page en appendice du chapitre décrivant cet indicateur ne comportant ni formule ni étapes de calcul, il est difficile de reproduire le score de démarchandisation et donc impossible de calculer les parts de chaque variable.
} 
auteurs ont fait le choix d'estimer le risque subjectif d'anxiété des individus à partir de quatre situations dont ils pouvaient évaluer schématiquement le coût moyen, ce qui les obligeait à faire quelques approximations et une hypothèse économique qu'ils ont formulée ainsi : «le changement de risque subjectif est supposé être proportionnel aux variations de risque objectif $»^{6}$. Le coût de la maladie a ainsi été estimé par les seules dépenses médicales non remboursées (ce qui laisse de côté le manque à gagner consécutif aux arrêts de travail); celui des pertes d'emploi a été obtenu à partir du taux de remplacement des indemnités de chômage ; celui du divorce et celui de la vieillesse à partir du taux de pauvreté moyen des mères seules et des personnes âgées ${ }^{7}$. Ces calculs ont l'avantage, moyennant quelques approximations $^{8}$, de procurer des estimations réalistes de l'importance des insécurités associées à quatre types de risque économique. Mais ils ne permettent pas d'évaluer l'ensemble des sécurités procurées par les prestations et aides garanties par l'État social.

Or, le développement des institutions de l'État social rend de plus en plus utile la disposition de concepts et d'outils statistiques permettant d'évaluer globalement le rôle des ressources indépendantes du marché, en particulier dans leur capacité à assurer à la population un minimum de sécurité, qui se trouve de ce fait «démarchandisée », elle aussi. C'est avec cet objectif en tête que nous avons cherché à construire un concept permettant d'approcher la sécurité protégeant les populations du risque de ne pas disposer de revenus suffisants, en raison par exemple des aléas de l'existence tels que le chômage, les accidents, les maladies ou les handicaps.

Pour ce faire, nous avons construit dès nos premiers essais un ensemble de conventions d'évaluation de la sécurité des populations (Menahem ; Cherilova, 2005), système que nous avons ensuite progressivement développé pour aboutir aux conventions actuelles (Menahem, 2007). En effet, comme le rappelle Jean Gadrey (2002) à propos de travaux sur les indicateurs, "on voit mal comment se passer de "conventions discutables", dès lors qu'il est question d'environnement, de qualité de vie et de progrès social, et donc de systèmes de valeurs. Même les indicateurs économiques "sérieux" sont truffés de conventions discutables, comme l'ont montré les débats sur les incertitudes très importantes des comparaisons du PIB par habitant en Europe ». Avant d'exposer les formules et les résultats de notre indicateur de "sécurité démarchandisée », nous allons donc énoncer l'état actuel des règles et conventions qui ont permis de les construire.

\section{Deux principes et trois types de conventions}

Deux principes sont à la base de la construction de notre indicateur. La sécurité doit d'abord être définie d'une manière relative, comme un rapport, afin de se référer au niveau de vie de la population environnante par rapport auquel un individu définit les besoins qu'il considère comme allant de soi. Deuxième principe, le sentiment de sécurité d'un individu est considéré comme une différence : différence entre le flux des sécurités associées aux diverses ressources indépendantes du marché dont il bénéficie, d'un coté, et le sentiment d'insécurité qui correspond aux manques dont il peut souffrir quand il veut se nourrir, se loger, se chauffer ou se vêtir, d'un autre coté.

Le caractère normatif des conventions permettant de mettre ces deux principes en pratique apparaît dans chacune des trois grandes catégories distinguées ci-après.

\footnotetext{
${ }^{6}$ Osberg ; Sharpe (2002), page 305.

${ }^{7}$ Ibidem, pages 306-308.

${ }^{8}$ Ces approximations non explicitées dans l'article cité concernent notamment les variations des probabilités qui ne peuvent être supposées additives (négatives pour l'insécurité et positives pour la sécurité) que moyennant des conditions concernant leur taille (qui doit être marginale) et leur indépendance mutuelle (qui doit autoriser que la probabilité des risques croisés soit égale au produit des probabilités).
} 
Les ressources concernées. Il nous fallait d'abord apprécier l'importance des ressources non associées directement à des échanges marchands et permettant, soit de suppléer à l'absence de rémunérations du travail, soit de compléter les salaires et revenus issus de l'activité professionnelle. Nous avons considéré que de telles ressources sont procurées par les revenus démarchandisés provenant des systèmes de protection sociale ou d'aide publique, indépendants par définition de liens directs de subordination aux employeurs ou aux marchés. Les individus perçoivent trois types de ces revenus tout au long de leur vie adulte, certains qu'ils aient un emploi ou non et d'autres parce qu'ils n'ont pas d'emploi.

- les remboursements et aides en nature : frais de maladie, charges familiales, aides au logement, ressources assurant une sécurité largement démarchandisée que les personnes soient en emploi ou sans emploi.

- les revenus de remplacement du travail associés aux risques vieillesse, chômage, maladie, invalidité ainsi que les pensions de réversion (veuvage).

- les indemnités et aides en nature mobilisées dans la lutte contre l'exclusion sociale (RMI, aides sociales, etc.).

La base de comparaison. Il nous fallait définir une référence par rapport à laquelle les habitants d'un territoire donné estiment leur niveau de vie et leurs besoins. Nous basant sur une analyse sociologique de la définition des besoins, nous avons considéré que ce sont les personnes ayant un emploi dans le pays qui jouent un rôle directeur et que leur revenu moyen est le plus légitime pour être considéré comme une référence. Cette convention est essentielle et obéit à une toute autre logique que celle établissant par exemple comme revenu de référence le revenu moyen (qui agrège les actifs et les inactifs) ou encore le PIB par habitant (plus représentatif du potentiel économique d'un pays que du niveau de vie). Elle aboutit dans les faits à un tout autre classement que ceux découlant des ratios classiques tels que le pourcentage des prestations sociales par rapport au PIB ou par rapport au Revenu National. ${ }^{9}$

En pratique, nous avons considéré que le revenu de référence par rapport auquel les individus évaluent leur sécurité est le niveau moyen du revenu disponible des actifs ayant un emploi, soit précisément la somme moyenne de leurs revenus nets d'impôts ou de cotisations (de l'activité professionnelle et de la propriété) et de leurs prestations famille, logement et maladie. Le premier élément positif du taux de sécurité nous est ainsi fourni par le «taux des ressources économiques démarchandisées», lequel est égal au rapport entre la somme des revenus démarchandisés et le revenu de référence dans le territoire considéré. (Voir annexe 2 pour le mode de calcul précis du numérateur et du dénominateur).

La pénalisation de l'insécurité économique. Une fois estimé le flux des sécurités positives, il nous fallait évaluer l'importance de l'insécurité économique qui peut en minorer ou annuler le bénéfice. Afin de prendre en compte l'insécurité économique première des personnes n'ayant pas les moyens de s'assurer les sécurités primordiales que sont les capacités à se nourrir, se loger, se chauffer et se vêtir, nous avons considéré que les ménages dont les ressources par tête sont inférieures ou égales au seuil de pauvreté dans le territoire considéré n'ont plus de sécurité : plus précisément, leur sécurité économique est d'un niveau d'autant plus négatif que leurs revenus sont plus inférieurs au seuil de pauvreté, soit $60 \%$ du revenu médian du pays selon les conventions d'Eurostat. Plusieurs outils mathématiques peuvent permettre de

\footnotetext{
${ }^{9}$ Par exemple, si nous comparons les estimations des prestations sociales relativement au PNB en 2002, telles celles publiées par Petràsova (2007) pour Eurostat, avec nos estimations des prestations relativement au revenu des personnes en emploi (voir les « taux de ressources démarchandisées » dans le tableau 1 suivant), nous constatons que la Norvège, les Pays-Bas et la Finlande reculent d'au moins 5 places si on les ordonne selon le PNB alors que la France, l'Allemagne et la Grèce voient leurs rangs progresser d'au moins 4 places dans le même cas.
} 
traduire cette convention. Nous avons choisi un moyen particulièrement simple : nous avons considéré que tous les adultes vivant dans un ménage pauvre (soit ne disposant pas de $60 \%$ du revenu médian) sont pénalisés par une insécurité négative valant $60 \%$ du revenu de référence, à savoir $60 \%$ du revenu disponible moyen des actifs ayant un emploi ${ }^{10}$, laquelle est déduite de leurs autres revenus démarchandisés.

Nous pouvons noter qu'une telle prise en compte de l'insécurité des adultes vivant dans des ménages pauvres fait indirectement intervenir le nombre des enfants qui vivent avec eux. Ainsi, la taille des ménages est un élément conditionnant de manière importante le niveau de l'insécurité économique dans un pays. Plus le nombre d'enfants des familles à faibles revenus est important, plus la proportion des adultes vivant dans des ménages pauvres est considérable et, en conséquence, plus l'insécurité économique pénalise le taux de sécurité démarchandisée.

Au niveau microéconomique, si nous considérons le taux de sécurité démarchandisée d'un adulte (Tsd) comme la différence entre son taux de ressources démarchandisées et l'insécurité économique qui peut le pénaliser du fait de son éventuelle situation de pauvreté, nous observons une discontinuité dans sa variation selon le niveau de revenu de son ménage $\mathrm{R}$. Dans l'exemple d'un adulte vivant dans un ménage comprenant $\mathrm{N}_{\mathrm{uc}}$ unités de consommation, si $\mathrm{Rm}$ est la partie marchandisée de son revenu et $\mathrm{Rd}$ sa partie démarchandisée, $R a$ étant le revenu disponible moyen des actifs en emploi et $R p$ le seuil de pauvreté (dont les niveaux sont donnés par ailleurs), son taux de sécurité démarchandisée Tsd peut prendre deux valeurs :

- $\mathrm{Si} \mathrm{R}=(\mathrm{Rm}+\mathrm{Rd}) / \mathrm{N}_{\mathrm{uc}}>R p$, alors on a $\mathrm{Tsd}=\mathrm{Rd} / R a$

- $\mathrm{Si} \mathrm{R}=(\mathrm{Rm}+\mathrm{Rd}) / \mathrm{N}_{\mathrm{uc}} \leq R p$, alors on a Tsd $=\left(\operatorname{Rd}-0,6^{*} R a\right) / R a=\mathrm{Rd} / R a-0,6$

Au niveau microéconomique, il y a bien une discontinuité de 0,6 dans le Tsd de l'individu quand le revenu de son ménage atteint le seuil de pauvreté $R p$. Au niveau macroéconomique, tout se passe comme si la population adulte en dessous du seuil de pauvreté se voyait affecté un revenu négatif d'un montant égal à $60 \%$ du revenu disponible moyen des personnes ayant un emploi. Cette forte pénalisation veut rendre compte du point de vue des populations sans ressources dont l'insécurité économique lamine les capacités de bien-être et de survie. Elle signale aussi la mise en cause des capacités des institutions ayant pour mission d'assurer aux individus une sécurité indépendante des aléas du marché. Au niveau global, elle doit donc être déduite de la sécurité démarchandisée procurée par les diverses institutions de l'État social.

Ce deuxième principe qui considère le sentiment de sécurité comme un solde manifeste l'importance que nous avons voulu donner à l'objectif de cohésion sociale, laquelle constitue, selon notre point de vue, un des éléments importants de la sécurité d'une population vivant dans un territoire donné. Si le TSD est très sensible à l'importance de la population pauvre, il l'est moins à celle des chômeurs et des retraités, si du moins la part maintenue au-dessus du seuil de pauvreté n'est pas modifiée quand elle varie ainsi que le niveau des allocations chômage ou retraite. Si, par exemple, la part des personnes âgées pauvres dans la population adulte reste constante, le TSD ne distingue alors pas l'effet du vieillissement qui accroît le nombre de retraités de l'effet de l'amélioration du niveau des retraites, lesquels augmentent

\footnotetext{
${ }^{10}$ Nous avons choisi ce pourcentage par référence à la définition du seuil de pauvreté dans les pays européens selon Eurostat. Dans la mesure où les personnes adultes vivant dans des ménages dont le revenu global par unité de consommation est inférieur à $60 \%$ du revenu médian sont considérées comme pauvre, il est logique d'évaluer leur insécurité économique par une valeur proche de ce minimum que l'institution statistique estime comme le minimum indispensable. Par souci de cohérence avec notre définition du dénominateur du revenu relatif, nous avons donc choisi d'estimer l'insécurité économique des ménages pauvres par $60 \%$ du revenu de référence. Le choix du revenu médian ou encore du revenu moyen comme revenu de référence aurait été également possible. Mais ce choix se serait référé à une autre logique.
} 
tous deux le niveau du TSD. De même, le TSD ne distingue pas les effets des variations du taux chômage et du niveau moyen des allocations si la part des chômeurs pauvres reste stable.

Au total, le taux de sécurité économique démarchandisé (TSD) est un indicateur relatif et différentiel. Il fait la somme des revenus issus de droits sociaux que les individus touchent tout au long de leur vie adulte dans le pays considéré et les divise par un dénominateur représentant le niveau de vie moyen des actifs ayant un emploi, déduction faite de l'insécurité subie par les ménages en situation de pauvreté.

\section{Les formules du TSD}

L'ensemble de ces conventions se traduit dans des formules comptables permettant de calculer et comparer les niveaux de sécurité démarchandisée des populations de divers pays. Nous pouvons les présenter sous deux formes plus ou moins agrégées. D'abord, à un niveau très macroéconomique, si nous appelons TSD le taux de sécurité démarchandisée et TIE le taux d'insécurité économique associée aux personnes vivant dans des ménages en dessous du seuil de pauvreté, nous avons :

TSD $=$ Revenus de remplacement + Remboursements + Aides \& indemnités contre l'exclusion - TIE Population adulte $*$ Revenu disponible moyen des actifs ayant un emploi

Avec TIE $=\underline{\text { Population pauvre }} * \underline{60 \% * \text { Revenu disponible moyen actifs employés }}$ Population adulte $\quad$ Revenu disponible moyen actifs employés

Une deuxième formule équivalente exprimant une approche partiellement désagrégée peut également être présentée. Nous pouvons décomposer la population adulte en trois catégories selon le type des revenus démarchandisés perçus : $1^{\circ}$ les individus qui touchent des revenus de remplacement (indemnités de chômage, d'invalidité, retraites et pensions de réversion); $2^{\circ}$ ceux qui touchent les aides et indemnités de lutte contre l'exclusion (minimums sociaux du type RMI, minimum vieillesse et autres indemnités dites «de solidarité») ; $3^{\circ}$ l'ensemble de la population (en emploi ou sans emploi) qui perçoit les revenus indirects correspondant aux remboursements des frais de maladie, allocations familiales et autres aides au logement. Sachant que le numérateur de la première formule peut être réparti entre les trois souspopulations et que chaque population doit être pondérée par son importance statistique, nous avons alors la formule suivante équivalente à la première :

$\begin{aligned} \mathrm{TSD}= & \% \text { Retraités, chômeurs \& invalides } * \frac{\text { Revenu moyen de remplacement (retraites \& allocations) }}{\text { Revenu disponible moyen des actifs en emploi }} \\ & +\% \text { Pauvres \& Exclus } * \frac{\text { Aides \& indemnités moyennes contre l'exclusion }}{\text { Revenu disponible moyen des actifs en emploi }} \\ & +100 \% \text { Population } * \frac{\text { Remboursements, allocations \& aides au logement }}{\text { Revenu disponible moyen des actifs en emploi }} \\ & -\% \text { Population pauvre } * \frac{60 \% * \text { Revenu disponible moyen actifs en emploi }}{\text { Revenu disponible moyen actifs en emploi }}\end{aligned}$

Les données. Pour examiner en quoi les concrétisations de ces formules théoriques permettent de rendre compte de la réalité des différences de sécurité entre les pays, nous avons eu recours aux bases de données conçues et rassemblées par Eurostat. Par là, nous avons hérité des catégories et nomenclatures que cet organisme européen a élaborées, en particulier à propos des prestations sociales, lesquelles sont enregistrées en huit groupes dans le système dit SESPROS (cf. Eurostat 1999). Enfin, même si Eurostat a à coordonner, contrôler et 
harmoniser les statistiques de l'Europe, le fait que les administrations des différents pays européens ne fournissent pas toutes les mêmes données, et pas en même temps, se traduit dans l'absence de certains chiffres sensibles pour certains d'entre eux et dans certains décalages (voir Annexe 1). Ceci explique que nous n'avons pu collecter les données nécessaires à l'évaluation du TSD que pour 19 des pays membres de l'Union européenne. Nous avons complété cette base par les données correspondant à la Norvège qui figurent également dans Eurostat depuis l'année 1995. Enfin la non disposition de données correspondant à nos décompositions nous a obligés à des approximations précisées dans l'Annexe 2 sur le mode de calcul du TSD.

Les résultats de nos évaluations témoignent de situations très inégales en Europe, par exemple entre les pays d'Europe du Nord et les pays d'Europe continentale et orientale. C'est ce que nous allons constater en analysant successivement la diversité des sécurités démarchandisées dans les vingt pays européens, puis leurs relations avec les niveaux de productivité et de production par personne.

\section{La diversité des ressources et des sécurités démarchandisées en Europe}

Les taux de sécurité démarchandisée (TSD) varient de manière considérable entre les 20 pays européens. C'est ce dont témoignent les estimations pour l'année 2002 issues des données d'Eurostat disponibles fin août 2006 et présentées dans le graphique 1. Nous avons vu que notre indicateur réalise une synthèse entre les ressources démarchandisées correspondant pour l'essentiel aux prestations sociales et le niveau de pauvreté monétaire qui y est associé. Or, pour chacune de ces variables, la distribution des pays selon le TSD donne pour l'essentiel des classements convergents. Il semble ainsi qu'une classification des États sociaux en quatre groupes découle de la prise en compte de la sécurité économique

\section{Quatre groupes d'États sociaux en Europe du point de vue de la sécurité démarchandisée}

Le graphique 1 sépare nettement, d'un côté, les pays d'Europe centrale et orientale (PECO) qui atteignent à peine un TSD de $6 \%$ et, de l'autre côté, les pays continentaux et le RoyaumeUni dont le TSD dépasse 13\%, puis les pays du Nord qui culminent au-delà de 22\%. Entre les deux, les pays du Sud dont les TSD s'étagent de 5\% à $11 \%$ semblent moins homogènes alors que pourtant ils sont proches tant par leur système de protection sociale que par leur traitement de la pauvreté. La signification de ces quatre groupes de pays peut être analysée sur les plans historique et institutionnel.

Les cinq «pays du Nord» apparaissent comme les plus homogènes. D'abord, ils se rattachent à la même tradition socio-démocrate d'intervention publique forte, de couverture universelle et de prestations généreuses, même s'ils n'ont pas affronté la crise des années 90 avec les mêmes réformes ${ }^{11}$. Le niveau de leur TSD témoigne des proximités de leur type d'État social : en 2002 , il varie entre $22 \%$ et $28 \%$ du revenu disponible moyen des actifs ayant un emploi. Le niveau de leurs ressources démarchandisées est le plus important (entre $29 \%$ et $34 \%$ du même revenu disponible moyen). Une autre homogénéité résulte à la fois de l'importance des prestations sociales et de la volonté des États socio-démocrates d'obtenir une forte cohésion sociale : le niveau de pauvreté monétaire est faible ; et il se traduit dans la modeste pénalisation correspondant à l'insécurité économique (de -6\% à -6,6\%).

\footnotetext{
${ }^{11}$ Voir le bilan complet des transformations de "l'État providence nordique" dans le numéro spécial de la Revue française des affaires sociales présenté par Strobel (2003) et regroupant les interventions à un colloque organisée par la MIRE.
} 
En revanche, le regroupement des dits «pays intermédiaires» est beaucoup plus hétérogène puisqu'il agrège quatre États dits «conservateurs» dans la typologie de Esping-Andersen (la Belgique, l'Allemagne, la France et l'Autriche) et un pays dit «libéral» (le Royaume-Uni) selon la même typologie avec un ancien pays socialiste avancé, la République Tchèque. Leurs TSD sont un peu plus dispersés puisqu'ils s'échelonnent entre $13 \%$ pour le Royaume-Uni et $22 \%$ pour l'Autriche. Un caractère commun les rassemble néanmoins : celui d'avoir un système de prestations sociales établi et développé depuis au moins un demi-siècle. Ceci se manifeste dans les niveaux importants de leurs ressources démarchandisées (allant de 23\% pour la Tchéquie à 30\% en Autriche). Il en découle des niveaux de la pauvreté monétaire et de l'insécurité économique associée plutôt faibles : de $-4,8 \%$ à $-9 \%$, sauf pour le Royaume-Uni dont le modèle libéral se traduisait en 2002 dans un niveau de $-10,8 \%{ }^{12}$.

\section{Graphique 1 : La diversité des taux de sécurité démarchandisée dans 20 pays européens en 2002}

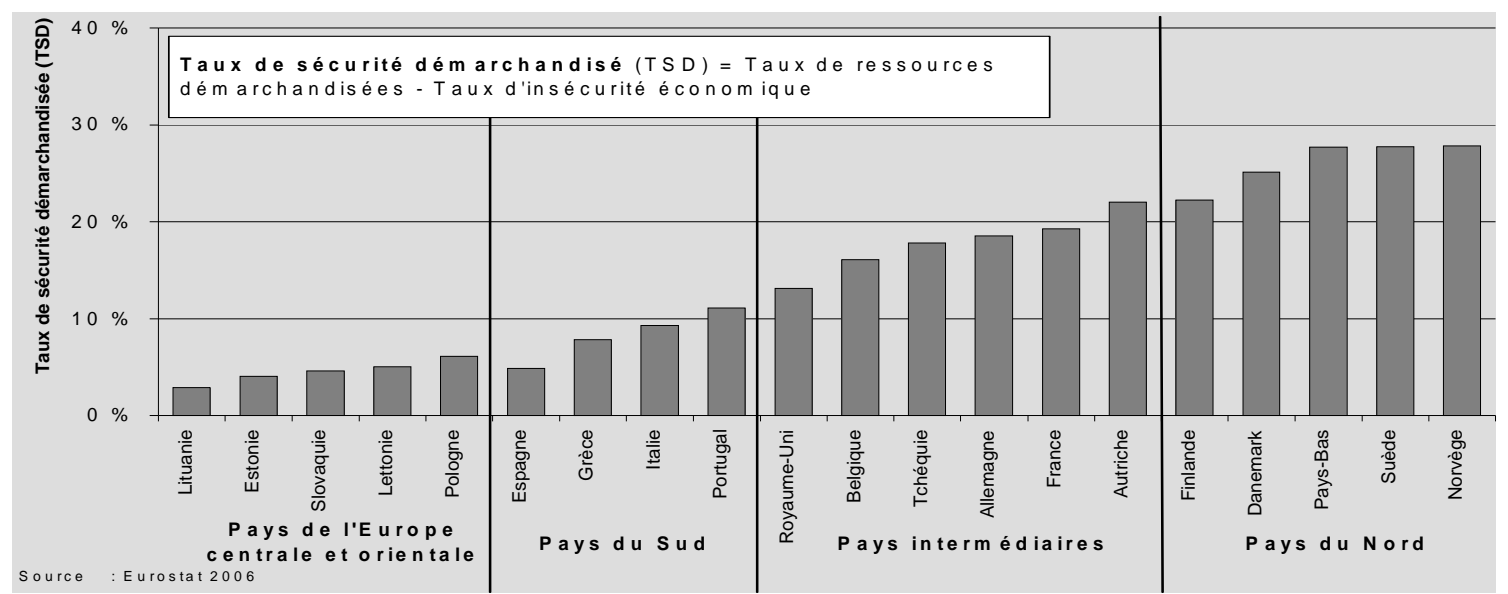

Source : Eurostat 2006

Lecture: le TSD de 2,9\% de la Lituanie est égal à la différence entre ses faibles ressources démarchandisées $(13,1 \%)$ et sa forte insécurité économique (-10,2\%). En France, en revanche, la plus faible insécurité $(-7,2 \%)$ se conjugue avec les plus importantes ressources démarchandisées (26,5\%), pour donner un TSD six fois supérieur (19,3\%).

Le groupe des pays «du Sud» rassemble de nombreux caractères communs. Le relativement faible niveau des prestations sociales s'explique par l'héritage de traditions clientéliste et corporatiste qui ont longtemps entraîné des obstacles aux réformes et au développement de l'État social. La Grèce, l'Italie, l'Espagne et le Portugal combinent ainsi chacun d'une façon particulière d'importants transferts financiers sur des bases corporatistes, la faiblesse des aides et services aux familles et démunis, et le développement de réseaux familiaux pour compenser ces insuffisances ${ }^{13}$. Cet héritage des quatre pays du Sud se manifeste dans le partage d'une même faiblesse des prestations sociales et donc des ressources démarchandisées : entre $20 \%$ et $23 \%$. Il entraîne également une même importance de la pauvreté monétaire et de

\footnotetext{
${ }^{12}$ Il faut noter toutefois le caractère surprenant de l'estimation du taux de pauvreté en Tchéquie, $8 \%$ soit $2 \%$ de moins que la Norvège, le pays nordique le mieux placé du fait de l'importance de ses prestations sociale. Eurostat a d'ailleurs émis des réserves sur cette estimation fournie par le gouvernement tchèque. Mais le bon classement du TSD de la Tchéquie se réfère aussi au niveau de ses ressources démarchandisées, bien plus élevé que dans les autres PECO. Une réévaluation du niveau de pauvreté de la Tchéquie à 10,8\% (soit $+35 \%$ ) ne diminuerait son TSD que de $1,7 \%$ (soit $-7,5 \%$ relativement), ce qui le mettrait au même niveau que la Belgique.

${ }^{13}$ Voir l'ensemble de textes rédigés à l'occasion des "Rencontres de Florence" organisée par la MIRE afin de

"Comparer les systèmes de protection sociale en Europe du Sud" avec leur conclusion (Rhodes ; Palier, 1997).
} 
l'insécurité économique correspondante : entre -11 et $-12 \%$. Ces deux caractères expliquent la faiblesse du TSD : entre 4,9\% pour l'Espagne et $11 \%$ pour le Portugal.

Le groupe «des PECO» (pays d'Europe centrale et orientale) présente aussi une homogénéité historique. Les cinq États sociaux correspondants sont tous issus d'un système socialiste ; ils ont engagé dans les vingt dernières années un même processus de transition vers l'économie de marché ; et ils se sont largement intégrés dans l'Union européenne. Bien que les histoires des redéfinitions de leurs prestations sociales soient elles aussi particulières, les ressources démarchandisées qui en résultent ont en commun leur faible niveau : de $13 \%$ à 17\% du revenu disponible moyen des actifs ayant un emploi. Les niveaux de pauvreté monétaire et l'insécurité économique associée sont donc importants : de $-10 \%$ en Lettonie à $-13 \%$ en Slovaquie. Il est alors logique de constater le très bas niveau des TSD de ces pays : entre $2,9 \%$ pour la Lituanie et $6,1 \%$ pour la Pologne.

Tableau 1 : Le classement et la composition des taux de sécurité démarchandisée dans 20 pays européens en 2002

\begin{tabular}{|c|c|c|c|c|c|c|c|c|c|}
\hline & $\begin{array}{l}\text { Données } \\
\text { Eurostat } 2002\end{array}$ & $\begin{array}{l}\text { Taux Ressources } \\
\text { Démarchandisées }\end{array}$ & $\begin{array}{l}\text { Rang } \\
\text { TRD }\end{array}$ & $\begin{array}{l}\text { Taux } \\
\text { pauvreté }\end{array}$ & $\begin{array}{l}\text { Rang Tx } \\
\text { Pvté }\end{array}$ & $\begin{array}{l}\text { Taux Insécurité } \\
\text { économique }\end{array}$ & $\begin{array}{l}\text { Rang } \\
\text { TIE }\end{array}$ & TSD & $\begin{array}{l}\text { Rang } \\
\text { TSD }\end{array}$ \\
\hline no & Norvège & $33,9 \%$ & 3 & $10,0 \%$ & 19 & $-6,0 \%$ & 2 & $27,9 \%$ & 1 \\
\hline se & Suède & $34,4 \%$ & 1 & $11,0 \%$ & 18 & $-6,6 \%$ & 3 & $27,8 \%$ & 2 \\
\hline $\mathrm{nl}$ & Pays-Bas & $34,3 \%$ & 2 & $11,0 \%$ & 16 & $-6,6 \%$ & 5 & $27,7 \%$ & 3 \\
\hline $\mathrm{dk}$ & Danemark & $31,7 \%$ & 4 & $11,0 \%$ & 15 & $-6,6 \%$ & 6 & $25,1 \%$ & 4 \\
\hline $\mathrm{fi}$ & Finlande & $28,8 \%$ & 6 & $11,0 \%$ & 17 & $-6,6 \%$ & 4 & $22,2 \%$ & 5 \\
\hline at & Autriche & $29,5 \%$ & 5 & $12,5 \%$ & 13 & $-7,5 \%$ & 8 & $22,0 \%$ & 6 \\
\hline $\mathrm{fr}$ & France & $26,5 \%$ & 8 & $12,0 \%$ & 14 & $-7,2 \%$ & 7 & $19,3 \%$ & 7 \\
\hline de & Allemagne & $27,5 \%$ & 7 & $15,0 \%$ & 11 & $-9,0 \%$ & 10 & $18,5 \%$ & 8 \\
\hline$c z$ & Tchéquie & $22,6 \%$ & 12 & $8,0 \%$ & 20 & $-4,8 \%$ & 1 & $17,8 \%$ & 9 \\
\hline be & Belgique & $24,5 \%$ & 9 & $14,0 \%$ & 12 & $-8,4 \%$ & 9 & $16,1 \%$ & 10 \\
\hline uk & Royaume-Uni & $23,9 \%$ & 10 & $18,0 \%$ & 7 & $-10,8 \%$ & 14 & $13,1 \%$ & 11 \\
\hline pt & Portugal & $23,1 \%$ & 11 & $20,0 \%$ & 3 & $-12,0 \%$ & 18 & $11,1 \%$ & 12 \\
\hline it & Italie & $20,7 \%$ & 13 & $19,0 \%$ & 5 & $-11,4 \%$ & 16 & $9,3 \%$ & 13 \\
\hline$g r$ & Grèce & $20,1 \%$ & 14 & $20,5 \%$ & 2 & $-12,3 \%$ & 19 & $7,8 \%$ & 14 \\
\hline es & Espagne & $16,3 \%$ & 17 & $19,0 \%$ & 4 & $-11,4 \%$ & 17 & $4,9 \%$ & 17 \\
\hline $\mathrm{pl}$ & Pologne & $16,3 \%$ & 16 & $17,0 \%$ & 9 & $-10,2 \%$ & 12 & $6,1 \%$ & 15 \\
\hline IV & Lettonie & $14,7 \%$ & 19 & $16,0 \%$ & 10 & $-9,6 \%$ & 11 & $5,1 \%$ & 16 \\
\hline sk & Slovaquie & $17,2 \%$ & 15 & $21,0 \%$ & 1 & $-12,6 \%$ & 20 & $4,6 \%$ & 18 \\
\hline ee & Estonie & $14,8 \%$ & 18 & $18,0 \%$ & 6 & $-10,8 \%$ & 15 & $4,0 \%$ & 19 \\
\hline It & Lituanie & $13,1 \%$ & 20 & $17,0 \%$ & 8 & $-10,2 \%$ & 13 & $2,9 \%$ & 20 \\
\hline
\end{tabular}

Source : Eurostat 2006

Lecture: la France est le septième pays selon le taux de sécurité démarchandisée (TSD) mais le huitième selon l'importance des ressources démarchandisées, ce qui est compensé par son taux de pauvreté relativement faible: sa pénalisation pour l'insécurité économique est au septième rang. La situation est inverse pour l'Allemagne, mieux placée pour les ressources démarchandisées mais plus défavorisée par la pénalisation associée à son taux d'insécurité plus élevé (au dixième rang).

Le tableau 1 résume la diversité des taux de sécurité démarchandisée pour les quatre groupes de pays européens. Il précise en même temps pour chacun des 20 pays considérés la synthèse que réalise le TSD ( $7^{\text {ème }}$ colonne de chiffres) entre les ressources démarchandisées $\left(1^{\text {ère }}\right.$ colonne) et le taux de pauvreté monétaire ( $3{ }^{\text {ème }}$ colonne), lequel est traduit dans une pénalisation, l'insécurité économique que les pauvres ont à supporter $\left(5^{\text {ème }}\right.$ colonne) et qui vient donc en déduction des ressources démarchandisées. Dans le tableau 1, les pays sont rangés dans l'ordre décroissant selon le niveau du TSD en 2002, à l'exception de l'Espagne qui a été remontée de trois places pour être classée avec les autres pays du Sud. Nous pouvons remarquer que les écarts de TSD entre les pays extrêmes de chaque groupe sont relativement 
faibles : 5,7\% pour les pays du Nord, mais 6,9\% pour les pays intermédiaires moins homogènes, 6,2\% pour les pays du Sud et 3,2\% pour les PECO. Ils reflètent essentiellement les écart des taux de ressources démarchandisées (respectivement 5,6\%;6,9\%; 4,5\% et $3,2 \%)$. En effet les taux d'insécurité économique sont beaucoup plus homogènes dans chaque groupe (d'écarts respectivement de $0,6 \% ; 3,6 \%{ }^{14} ; 0,9 \%$ et $3 \%$ ).

Cette importante diversité des niveaux de sécurité économique correspond également aux types des politiques sociales adoptées dans les différents pays. Comment en effet ne pas remarquer que les TSD les plus élevés s'observent dans les pays du Nord, là où les compromis noués avec les syndicats ont abouti à institutionnaliser un haut niveau de protections sociales ? Si les revenus indépendants du marché du travail y sont plus importants, c'est parce que les politiques sociales visent davantage à l'intégration des personnes du fait des droits issus de leur citoyenneté. En même temps, les politiques sociales y multiplient les aides et services en nature, ce qui concourt à la limitation des situations de pauvreté et donc de l'insécurité économique. En revanche, au Royaume-Uni et au Portugal, là où la sécurité dépend plus du marché du travail et où les prestations sociales se limitent davantage à un mince filet de sécurité, les proportions de pauvres sont plus importantes, et donc aussi les pénalisations associées de l'insécurité : de $11 \%$ à $14 \%$.

\section{La disparité des évolutions des taux de sécurité démarchandisée de 1995 à 2003}

Comme en témoigne la situation actuelle des PECO, la construction des systèmes de protection sociale est un long processus. Un autre article serait nécessaire pour analyser d'une manière comparative les différents cheminements qui ont permis d'aboutir aux hauts niveaux actuels de sécurité économique des pays du Nord ou des pays intermédiaires. Si nous nous limitons ici aux enseignements fournis par les données d'Eurostat, nous pouvons observer les séries des différentes ressources démarchandisées et des taux de pauvreté pour certains pays entre 1995 et 2003. Nous pouvons en déduire l'évolution des taux de sécurité démarchandisée pour cette période. Le graphique 2 illustre la diversité de ces variations du TSD et différencie chacun des quatre groupes de pays par un style de trait particulier, ce qui permet de visualiser par exemple la progression plus rapide des taux de l'ensemble des pays du Sud.

Là encore, ces variations recouvrent des phénomènes différents. D'un côté, les évolutions des prestations sociales et des ressources démarchandisées correspondantes sont dépendantes à court terme des mouvements de la conjoncture, notamment pour les allocations chômage et les aides à la lutte contre l'exclusion, et aussi, à long terme, du gonflement des multiples prises en charge associées au vieillissement de la population. Mais, d'un autre côté, l'insécurité économique est sensible aussi à la conjoncture économique, directement du fait des liens des taux de pauvreté avec le chômage et plus indirectement du fait de sa relation avec le mouvement de précarisation des emplois.

Par exemple, le Portugal donne l'exemple vertueux d'un pays qui, partant d'une situation pour le moins rudimentaire en matière sociale, développe son système de prestations tout en limitant quelque peu le taux de pauvreté préoccupant de la fin des années 1980. Résultat, entre 1996 et 2003, à l'exception d'une pause en 2001, les ressources démarchandisées ont augmenté régulièrement, de $18 \%$ à $25 \%$ du revenu disponible moyen des personnes ayant un emploi, alors que dans le même temps l'insécurité économique diminuait de $13 \%$ à $11 \%$. Ceci explique la remarquable progression de son TSD qui a été multiplié par 2,6 en 8 ans. Toutefois, dans les autres pays du Sud, le rythme encore plus rapide des progressions n'est pas associé à des diminutions de la pauvreté. Il vient du fait que les TSD initiaux étaient très

\footnotetext{
${ }^{14} \mathrm{Si}$ l'on met à part le cas de la Tchéquie pour laquelle nous avons rappelé plus haut les réserves d'Eurostat.
} 
limités en 1995 (entre 1,5\% et 2,4\%), ce qui a démultiplié l'importance de l'augmentation des prestations sociales qui ont suivi les réformes et les luttes sociales.

\section{Graphique 2 : La diversité des évolutions des taux de sécurité démarchandisée dans 20 pays européens entre 1990 et 2003}

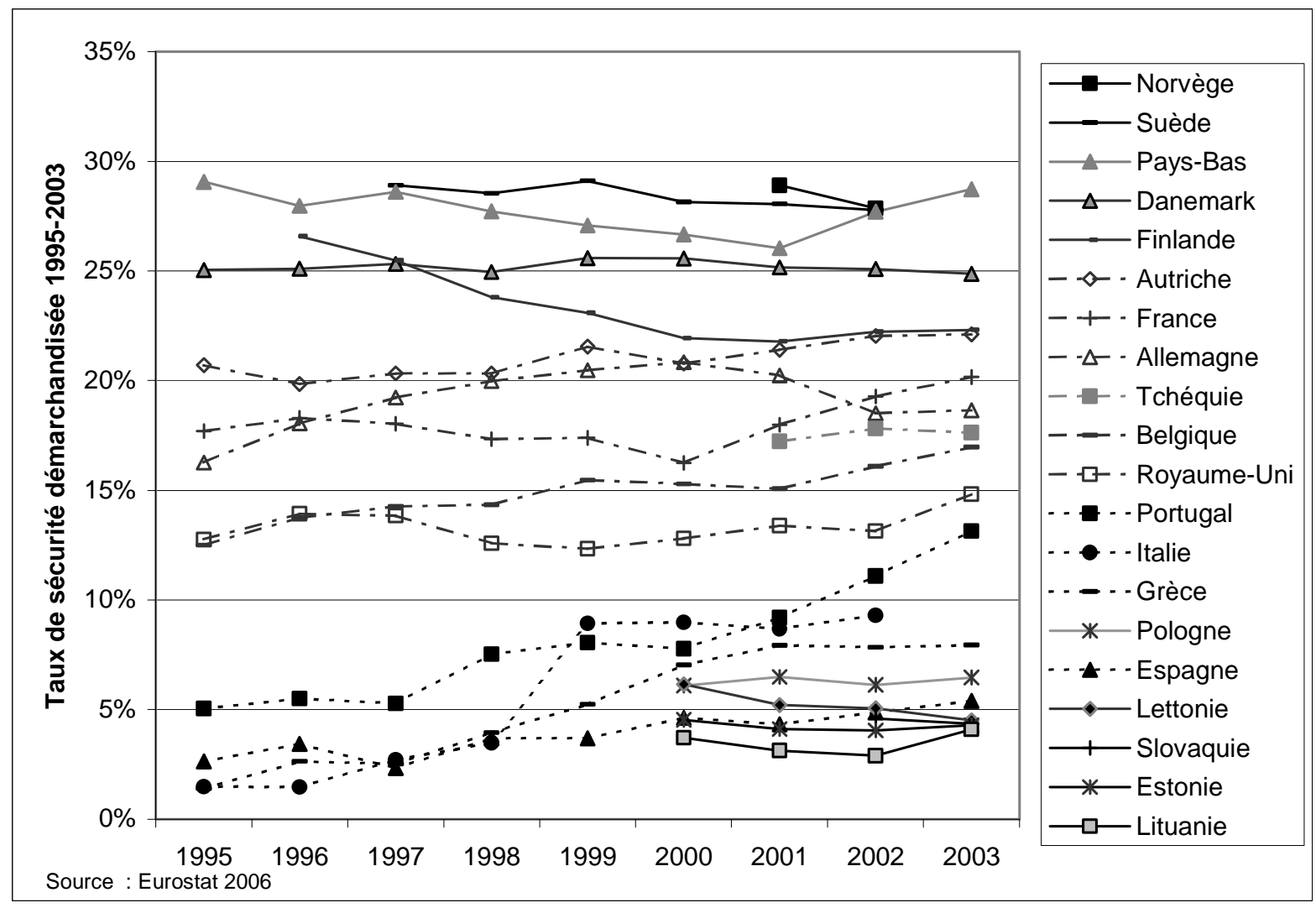

Lecture : Le taux de sécurité démarchandisée des Pays-Bas diminue de 1995 à 2001, passant de 29\% à $26 \%$ pour remonter à $28,7 \%$ en 2003, soit presque à sa valeur de départ. En revanche le TSD du Portugal augmente continûment de 1995 à 2003, s'élevant d'un niveau de 5\% à $13 \%$.

Dans les pays du Nord et les pays intermédiaires, les évolutions des ressources démarchandisées ont été plus chaotiques et, en tout cas, beaucoup moins profitables à la sécurité des populations. Le déclin ou la stagnation des prestations sociales observé entre 1995 et 2002 en Finlande, en Suède et aux Pays-Bas a coïncidé avec la stabilité du taux de pauvreté et donc de l'insécurité économique, ce qui s'est traduit par un léger tassement des TSD des pays considérés, mais certes à un haut niveau - deux ou trois fois supérieur à celui des pays du Sud. Dans cet arrière-plan morose, l'exception de la France des années 2000 à 2003 mérite d'être notée. Ses ressources démarchandisées augmentent (de 25,9\% à 26,4\%) dans le même temps que son taux de pauvreté diminue. Ce double mouvement a entraîné une augmentation notable de son TSD (de 16\% à 20\%) alors même qu'il diminuait en Allemagne (de $21 \%$ à $18 \%$ ) pour des raisons inverses.

Mais la relation entre le niveau de sécurité économique et le niveau de la production prendelle toujours le même sens ? Est-ce toujours l'abondance des ressources qui permet aux gouvernements de financer des systèmes plus généreux de protection sociale ? N'existe-t-il pas des mouvements en retour qui permettraient de comprendre que davantage de sécurité économique produise plus de cohésion sociale, laquelle favoriserait la productivité et la croissance ? Ce sont ces autres relations que nous allons explorer maintenant. 


\section{La diversité des associations de la sécurité avec la productivité et le niveau de vie dans les pays européens}

Eurostat diffuse des normes et consignes conduisant au rassemblement de statistiques de productivité et de production dites en SPA (Standard de Pouvoir d'Achat), c'est à dire dans une monnaie commune qui réduit fortement l'incidence des variations des prix et de taux de change entre les pays. Ces données permettent de comparer les volumes de production de chaque pays et aussi, en les divisant par le nombre d'heures travaillées, les productivités horaires de leurs travailleurs. En croisant ces données avec celles que nous avons construites en matière de sécurité économique, nous avons constaté qu'elles étaient fortement corrélées. Ce sont ces graphiques et les associations qu'elles mettent en évidence que nous allons successivement commenter sur les données de 2002 (plus complètes que celles de 2003).

\section{Les associations des niveaux de productivité horaire avec les taux de sécurité démarchandisée}

Le graphique 3 croise, pour chacun des 20 pays européens, le niveau de productivité par heure travaillée avec le taux de sécurité démarchandisée que nous avons précédemment défini. Il est remarquable de constater d'abord la forte corrélation de cet ensemble de données $(0,76)$. Tout se passe comme si, plus la sécurité de la population s'accroissait, plus la productivité horaire des travailleurs augmentait. Mais il faut examiner de près cette hypothèse.

Graphique 3 : La diversité des associations des niveaux de productivité horaire avec la sécurité démarchandisée dans 20 pays européens en 2002

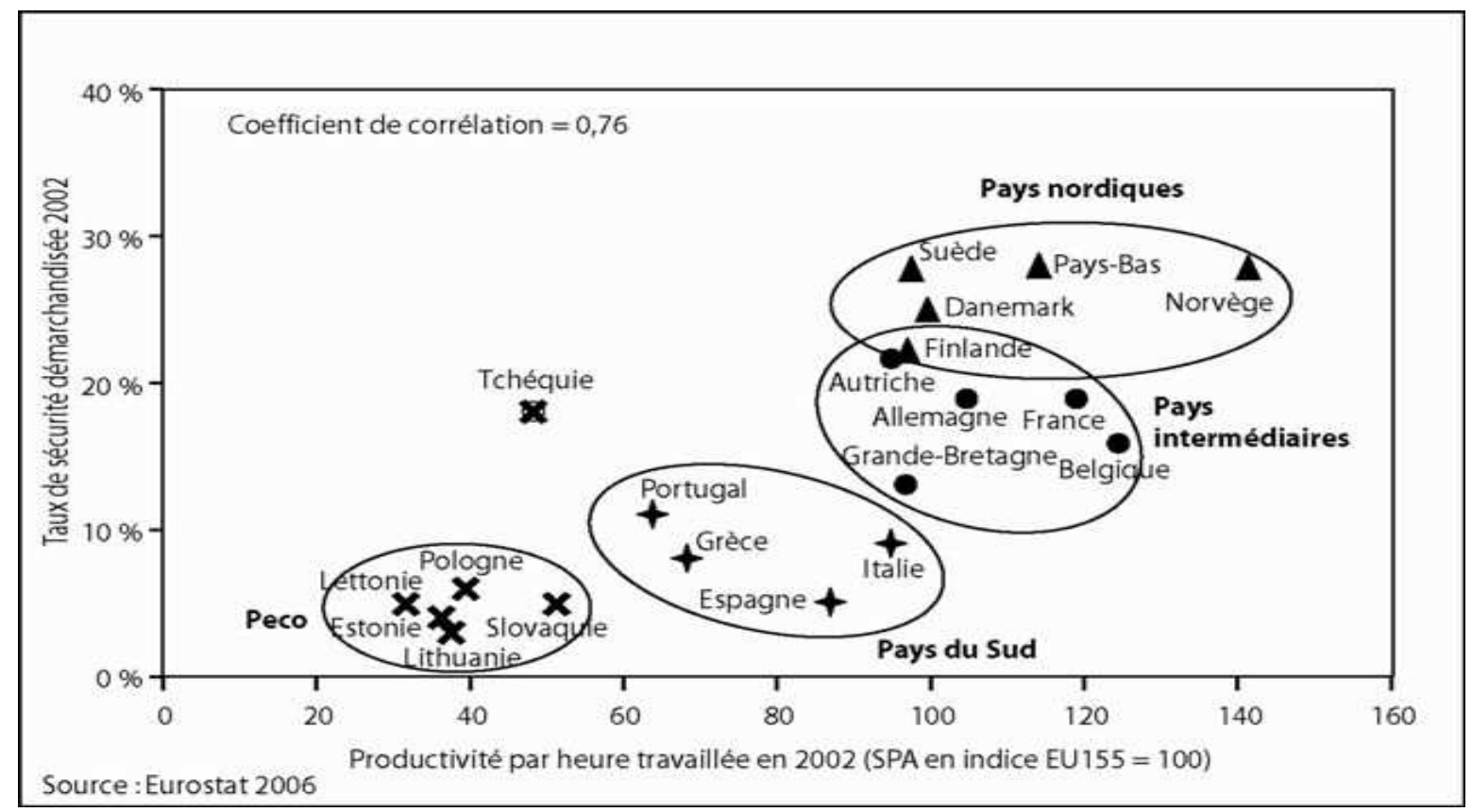

Lecture: dans le groupe des pays dits "intermédiaires", le taux de sécurité démarchandisée est compris entre $13 \%$ et $22 \%$ et la productivité par heure travaillée varie entre les indices 95 et 125, 100 représentant la moyenne de l'Europe des 15. La France y occupe une position privilégiée : deuxième pays selon le taux de sécurité démarchandisée, après l'Autriche, et deuxième aussi selon la productivité horaire, à 119, derrière la Belgique.

Que se passe-t-il pour les quatre groupes de pays dont nous avons synthétisé précédemment les caractéristiques ? Le graphique 3 montre qu'ils restent homogènes aussi selon la productivité horaire. L'opposition est très nette entre, d'un côté, le groupe des PECO dont la productivité varie faiblement autour d'un tiers du niveau de l'Europe des 15 (entre les indices 32 et 40, à l'exception de la Slovaquie à 51) et, de l'autre côté, les deux groupes de pays 
développés, du Nord et intermédiaires, dont le niveau se situe autour de la moyenne de l'Europe ou bien au-delà (entre 95 et 141). Entre les deux, le groupe des pays du Sud manifeste les différences de développement opposant le Portugal (64) et l'Italie (95). Enfin, il faut noter que la Tchéquie que nous avions regroupé avec les pays intermédiaires pour son système social se retrouve, pour sa productivité horaire (à l'indice 48) au niveau de la Slovaquie (51), ce qui empêche de l'intégrer à un groupe pour ces deux dimensions.

Comment interpréter ces proximités? Intuitivement, nous pouvons nous dire que plus la population est en sécurité du point de vue des revenus, plus les personnes peuvent envisager des projets d'avenir, plus la cohésion sociale est grande et plus les travailleurs peuvent se sentir motivés pour déployer leurs compétences et talents. C'est un peu cette interprétation que proposaient les experts du Forum de Davos quand, commentant leur classement mondial 2005 des pays selon des critères de pure compétitivité économique, ils écrivaient : «Rien ne prouve que l'ampleur des prélèvements fiscaux effectués dans les pays nordiques les empêche d'offrir à leur population l'un des niveaux de vie les plus élevés de la planète. [...] De fait, ce sont ces mêmes prélèvements et les recettes publiques qu'ils génèrent qui ont permis de créer des établissements scolaires de premier plan, d'instaurer un système de sécurité sociale exemplaire ou d'alimenter le marché du travail avec une main-d'œuvre hautement qualifiée et ultra-motivée ${ }^{15}$.

Dans un deuxième temps, nous pouvons nous demander s'il n'y a pas un facteur explicatif commun à ces corrélations. Par exemple, quand un pays est plus riche en terme de produit par habitant, cela exprime d'un côté qu'il a accumulé plus de savoir-faire et de dispositifs techniques hautement productifs, ce qui permet de comprendre la liaison avec la productivité horaire. Mais cela manifeste aussi, d'un autre côté, qu'il a plus les moyens de consacrer des fonds importants aux prestations sociales et aux actions contre les inégalités, d'où un taux de pauvreté plus faible et une sécurité plus grande. Il en résulte une association indirecte entre productivité et sécurité. Ce raisonnement est valable à la fois pour les PECO, en négatif, et pour la Norvège, les Pays Bas ou la France, en positif. Mais il permet mal de comprendre, par exemple, les cas spécifiques de l'Espagne (productivité horaire moyenne pour un bas taux de sécurité démarchandisée) ou de la Tchéquie (faible productivité horaire avec un taux de sécurité moyen). Nous allons retrouver ces questions, mais un peu atténuées, dans le cas de la production par habitant.

\section{Les associations des niveaux de production par tête avec les taux de sécurité démarchandisée}

Le graphique 4 résulte lui du croisement, pour chacun des 20 pays européens, du taux de sécurité démarchandisée et du niveau de production par habitant (en indice, la moyenne de l'Europe des 15 valant 100). La corrélation de ces données $(0,82)$ est encore plus forte qu'avec la productivité horaire. En effet, les groupes de pays apparaissent plus compacts dans le graphique 4 que dans le graphique 3 : les pays du Nord et les pays intermédiaires sont en particulier bien moins étalés. Seul le groupe des pays du Sud semble toujours être très étendu entre les niveaux de vie italien (indice 107) et ceux du Portugal ou de la Grèce (76). Enfin, la Tchéquie reste encore dans une situation inclassable, son TSD la rattachant plus aux pays

\footnotetext{
${ }^{15}$ World Economic Forum (2005), Le Forum Économique Mondial de Davos a surpris en plaçant deux pays européens du Nord, la Finlande et le Danemark, aux deuxième et troisième places de son classement 2005 de la compétitivité. D'où le besoin d'expliquer leur succès. Nous pouvons noter de plus que la Suède est au septième rang et les Pays-Bas à la onzième place. Quant à la Norvège, il est surprenant de ne la trouver qu'au $16^{\text {ème }}$ rang, alors que nous avons vu que sa productivité horaire et son PIB par habitant la plaçaient nettement devant les autres pays européens, en 2002 il est vrai. Mais les managers et experts interviewés par le WEF font intervenir certainement de multiples autres critères de classement.
} 
intermédiaires alors que son niveau de vie, certes supérieur à celui des PECO, est inférieur à celui des pays du Sud ${ }^{16}$.

\section{Graphique 4 : La diversité des associations des niveaux de production par habitant avec la sécurité démarchandisée dans 20 pays européens en 2002}

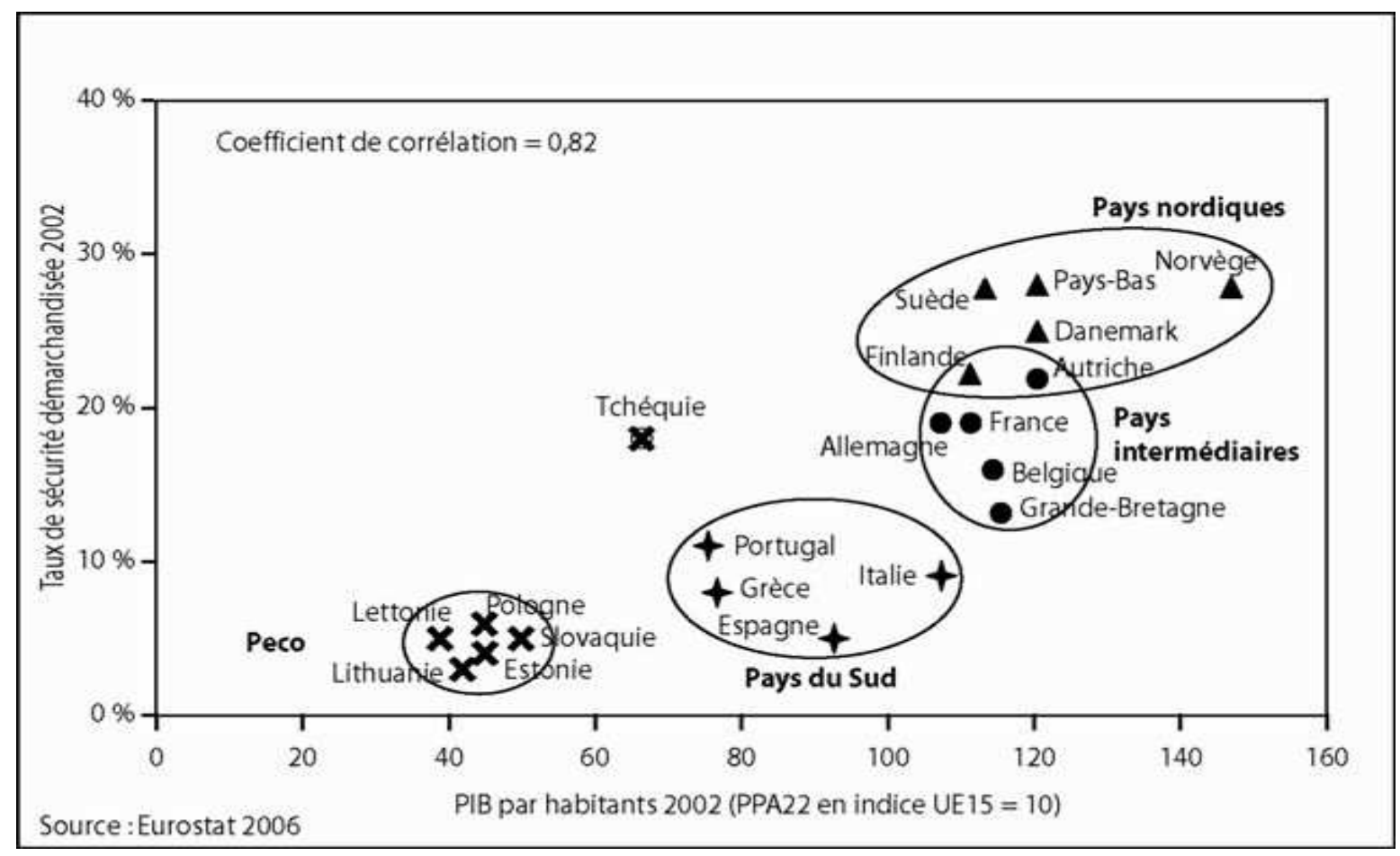

Lecture: dans le groupe des pays du Nord, le taux de sécurité démarchandisée varie entre $22 \%$ et $28 \%$ et la production par habitant est comprise entre les indices 111 et 147, 100 représentant la moyenne de l'Europe des 15. La Norvège y réalise à la fois le meilleur niveau de production par habitant et le meilleur taux de sécurité démarchandisée, pratiquement à égalité avec les Pays-Bas et la Suède.

En Europe, la sécurité augmente ainsi avec le niveau de vie et, réciproquement, les taux de sécurité sont plus importants là où le PIB par habitant est plus considérable. Ce qui apparaît comme le plus important dans ce résultat n'est sans doute pas la corrélation globale, mais plus la mise en évidence de groupes de pays homogènes, dans lesquels les faibles ou hauts niveaux de sécurité démarchandisée correspondent avec d'également faibles ou hauts résultats économiques au sens classique, en matière de production ou de productivité horaire. La prise en considération des caractères de ces groupes de pays conduit à proposer des interprétations des différences de croissance qui divergent fortement des interprétations en terme de « charges sociales excessives ».

Pour relativiser les idées simples concernant le rôle des prestations sociales en matière de croissance et de niveau de vie

Nous venons de constater que les prestations sociales, la lutte contre la pauvreté, et la sécurité démarchandisée qui en découle, sont associées fortement aux niveaux de productivité. Avec de multiples autres facteurs, ils contribuent à expliquer la bonne santé de l'économie. L'ampleur excessive des prestations et celle des impôts qui leur sont associés pourraient-elles

\footnotetext{
${ }^{16}$ La réévaluation de $+35 \%$ de son taux de pauvreté, telle que nous l'avons proposée dans la note 12 , entrainerait une diminution de l'estimation de son TSD à 16,1\%, le niveau de la Belgique. Elle n'atténuerait donc que marginalement ce caractère inclassable.
} 
expliquer alors les niveaux de croissance insuffisants de certains pays européens ? Nous avons rappelé dans l'introduction combien ces thèses ont de l'influence. Or l'examen des données de Eurostat présentées dans le tableau 2 met en cause la portée de telles explications par le rôle semble-t-il plus considérable de l'impact des conditions initiales : le taux de croissance serait d'autant plus important que le retard des niveaux de vie était plus grand au départ. C'est ce facteur qui semble avoir joué un rôle déterminant dans le cas des PECO en 1994, ou encore pour la Finlande et la Suède qui, en 1993, sortaient à peine de la crise bancaire scandinave.

Le tableau 2 introduit en effet des données permettant de comparer les corrélations des taux de croissance moyens de 1995 à 2003 avec les deux types de déterminants : d'une part les prestations sociales et les niveaux d'impôt par habitant, qui auraient été moteurs en 2002 ainsi que le suggère les analystes de la Commission européenne (2004); d'autre part les niveaux du PIB par habitant et de consommation finale par personne en 1995 dans les 20 pays européens considérés $^{17}$, déterminants qui peuvent suggérer d'autres interprétations.

Dans le tableau 2, les pays sont classés selon l'évaluation de la sécurité démarchandisée par le TSD, ce qui fait apparaître les quatre types dont nous avons mis en évidence les caractères. Les deux dernières colonnes indiquent notre variable à expliquer, à savoir le taux moyen de croissance du PIB entre les années 1995 et 2003 et le rang respectif des pays selon ce critère. Les comparaisons avec les rangs du TSD, du PIB ou de la consommation finale par habitant montrent des corrélations inverses avec les taux moyens de croissance. Pour aller plus loin dans l'analyse, nous avons été conduits à recourir à des exploitations statistiques complémentaires.

- Les calculs des coefficients de corrélation des taux de croissance avec ces diverses variables ont d'abord été très utiles car ils permettent de prendre en considération l'ensemble des proximités ou écarts entre les séries prises deux à deux.

- Les régressions linéaires du type «taux de croissance = f(Prestations 2002, PIB 1995) », ont fourni de plus des indications sur les corrélation multiples avec d'autres variables, ce qui a donné d'autres moyens pour interpréter la variabilité des taux de croissance ${ }^{18}$.

Cinq types de résultats significatifs s'en dégagent.

\footnotetext{
${ }^{17}$ Ces différentes données sont évaluées en SPA, monnaie qui permet de faire abstraction des différences de taux de change et de variation des prix. Si nous tentons des calculs de corrélation avec des séries en euros courants non standardisées, nous constatons des niveaux de corrélation bien moins importants, avec les prestations, les impôts ou les indicateurs de niveau de vie. De même pour des données non calculées par habitant.

${ }^{18}$ Même avec notre petit échantillon de 20 pays, les estimations des modèles avec 2, 3 ou 4 variables explicatives sont toujours globalement significatives. Les «valeurs critiques du F de Fisher » varient respectivement entre $3 * 10^{-6}$ et $1,4 * 10^{-4}$, ce qui rend les estimations des modèles largement satisfaisantes au seuil de $1 \%$.
} 
Tableau 2 : Comment interpréter la diversité des taux de croissance dans 20 pays européens ? Par les prestations sociales ? Par les impôts? Ou par le rattrapage du niveau de vie ?

\begin{tabular}{|c|c|c|c|c|c|c|c|c|c|c|c|}
\hline \multirow[t]{2}{*}{$\begin{array}{l}\text { Vingt pays } \\
\text { ordonnés } \\
\text { selon le TSD }\end{array}$} & \multirow{2}{*}{$\frac{T S D}{2002}$} & \multicolumn{2}{|c|}{$\begin{array}{l}\text { Prestations sociales } \\
\text { SPA par habitant }\end{array}$} & \multicolumn{2}{|c|}{$\begin{array}{c}\text { Impôts } \\
\text { SPA par habitant }\end{array}$} & \multicolumn{2}{|c|}{$\begin{array}{c}\text { PIB } \\
\text { SPA par habitant }\end{array}$} & \multicolumn{2}{|c|}{$\begin{array}{l}\text { Consommation finale } \\
\text { SPA par habitant }\end{array}$} & \multicolumn{2}{|c|}{$\begin{array}{c}\text { Taux de } \\
\text { croissance moyen } \\
95-03\end{array}$} \\
\hline & & 1995 & 2002 & 1995 & 2002 & 1995 & 2002 & 1995 & 2002 & $\%$ & rang \\
\hline Norvège & $27,9 \%$ & 5271 & 8106 & 2800 & 3900 & 20100 & 31600 & 9900 & 14100 & 3,0 & 10 \\
\hline Suède & $27,8 \%$ & 6148 & 7609 & 2400 & 3100 & 18100 & 24500 & 8900 & 12000 & 2,9 & 13 \\
\hline Pays-Bas & $27,7 \%$ & 5350 & 6978 & 1800 & 2900 & 18400 & 27000 & 9100 & 13500 & 3,0 & 11 \\
\hline Danemark & $25,1 \%$ & 5953 & 7546 & 2600 & 3800 & 19100 & 26100 & 9800 & 12400 & 2,1 & 18 \\
\hline Finlande & $22,2 \%$ & 4966 & 6137 & 2000 & 3100 & 16100 & 24700 & 8400 & 12400 & 3,7 & 8 \\
\hline Autriche & $22,0 \%$ & 5506 & 7329 & 2000 & 2600 & 19500 & 25800 & 11100 & 14600 & 2,2 & 16 \\
\hline France & $19,3 \%$ & 5091 & 6860 & 1900 & 2400 & 17600 & 24100 & 10000 & 13500 & 2,3 & 15 \\
\hline Allemagne & $18,5 \%$ & 5060 & 6763 & 1800 & 2300 & 18500 & 23400 & 10700 & 13800 & 1,4 & 20 \\
\hline Tchéquie & $17,8 \%$ & 1805 & 2851 & 1000 & 1300 & 10600 & 14600 & 5400 & 7500 & 2,2 & 17 \\
\hline Belgique & $16,1 \%$ & 4872 & 6759 & 1900 & 2700 & 18700 & 25300 & 10100 & 13500 & 2,4 & 14 \\
\hline Royaume-Uni & $13,1 \%$ & 4487 & 6394 & 1800 & 2800 & 16700 & 25000 & 10600 & 16500 & 2,9 & 12 \\
\hline Portugal & $11,1 \%$ & 2236 & 3718 & 1400 & 2200 & 11600 & 17100 & 7600 & 10800 & 3,3 & 9 \\
\hline Italie & $9,3 \%$ & 4227 & 5791 & 1800 & 2400 & 18100 & 23700 & 10500 & 13900 & 1,6 & 19 \\
\hline Grèce & $7,8 \%$ & 2367 & 4246 & 1100 & 2000 & 10900 & 16600 & 8000 & 11400 & 3,8 & 7 \\
\hline Espagne & $4,9 \%$ & 2838 & 3918 & 1000 & 1900 & 13500 & 20500 & 8100 & 11900 & 3,8 & 6 \\
\hline Pologne & $6,1 \%$ & nd & 2072 & 700 & 1200 & 6300 & 10000 & 3800 & 6700 & 4,3 & 4 \\
\hline Lettonie & $5,1 \%$ & nd & 1113 & 600 & 800 & 4600 & 8300 & 2900 & 5200 & 5,8 & 3 \\
\hline Slovaquie & $4,6 \%$ & 1229 & 2024 & 800 & 1100 & 6900 & 11000 & 3600 & 6400 & 4,2 & 5 \\
\hline Estonie & $4,0 \%$ & nd & 1284 & 600 & 1100 & 5200 & 10100 & 2900 & 5700 & 6,6 & 1 \\
\hline Lituanie & $2,9 \%$ & nd & 1235 & 600 & 1000 & 5300 & 9000 & 3500 & 5800 & 6,0 & 2 \\
\hline \multicolumn{2}{|c|}{$\begin{array}{l}\text { Coefficients de corrélation av. } \\
\text { taux de croissance moyen }\end{array}$} & $\begin{array}{l}-0,484 \\
-0,484\end{array}$ & $\begin{array}{l}-0,781 \\
-0,479\end{array}$ & $\begin{array}{l}-0,707 \\
-0,404\end{array}$ & $\begin{array}{l}-0,633 \\
-0,209\end{array}$ & $\begin{array}{l}-0,838 \\
-0,607\end{array}$ & $\begin{array}{l}-0,748 \\
-0,389\end{array}$ & $\begin{array}{l}-0,842 \\
-0,600\end{array}$ & $\begin{array}{l}-0,770 \\
-0,405\end{array}$ & \multicolumn{2}{|c|}{$\begin{array}{l}\text { Pour les } 20 \text { pays } \\
\text { Sans } 4 \text { PECO }\end{array}$} \\
\hline
\end{tabular}

Source : Eurostat 2006

Lecture : la série des taux moyens de croissance est inversement corrélée avec la série des impôts par habitant, davantage avec celle de l'année 1995 (-0,707) qu'avec celle de 2002 (-0,633); elle l'est encore plus avec la série des PIB par habitant de 1995 (-0,838) et davantage encore avec celle des consommations finales de 1995 (-0,842). La série des prestations sociales par habitant relative à 2002 semblerait être plus corrélée avec la croissance moyenne, mais à un plus faible niveau (-0,781). Ce conditionnel vise à souligner que, pour les prestations, les coefficients pour 2002 et 1995 ne sont pas comparables : le dernier ne concerne qu'une série sans les PECO. La dernière ligne montre que les corrélations des séries sans les quatre PECO non renseignés sont toutes plus faibles en 2002 que celles concernant 1995, et à un bien plus faible niveau. Voir notes 19 et 20 pour plus de détails.

1. Les taux de croissance des 20 pays européens sont plus corrélés avec les niveaux de vie des années de départ qu'avec les prestations ou les impôts. C'est d'abord le niveau de vie en 1995 et non pas celui de 2002 qui est le plus corrélé avec les taux de croissance moyens, tant pour le PIB par habitant que pour le niveau de consommation finale des ménages et des institutions sans but lucratif ${ }^{19}$, la consommation finale étant un peu plus explicative.

* Nous constatons ensuite que les niveaux de corrélation inverse des taux moyens avec les prestations par habitant en 2002 ou les impôts par habitant en 1995 sont d'un niveau plus faible qu'avec l'un ou l'autre indicateur du niveau de vie du pays en 1995 (-0,781 et $-0,707$ contre $-0,838$ ou $-0,842$ ).

* Nous observons de plus que les impôts par habitant de 1995 sont plus corrélés avec les taux de croissance moyens $(-0,707)$ que les impôts de $2002(-0,633)$, ce qui plaide moins pour la thèse du rôle déterminant de la contrainte de la pression fiscale en 2002 et davantage pour celle du primat du retard en 1995 du niveau de vie dans l'explication de la diversité des taux

\footnotetext{
${ }^{19}$ Le calcul des coefficients de corrélation avec les taux moyens de croissance pour l'ensemble des années 1995 à 2003 montre qu'ils diminuent régulièrement, à deux petites exceptions près, de l'année de départ à l'année d'arrivée. Par exemple, pour la consommation finale SPA, nous observons une diminution régulière des corrélations de -0.842 pour 1995 , puis $-0.838,-0.833,-0.822,-0.816,-0.800,-0.790,-0.770$ et -0.754 pour 2003. De même, pour les impôts SPA par habitants, nous constatons une baisse tendancielle des corrélations de -0.707 pour 1995, puis $-0.683,-0.645,-0.654,-0.664,-0.659,-0.634,-0.633$ et -0.604 pour 2003.
} 
de croissance (le poids des impôts 1995 reflétant plus le niveau de la consommation en 1995). Le fait que la corrélation négative du niveau des impôts avec la croissance ne soit plus significative lorsque nous prenons en compte d'autres variables montre également qu'il faut l'attribuer en premier lieu à la pression du rattrapage des niveaux de vie.

* Quant à la série des prestations sociales, si nous enlevons les PECO qui ne sont pas renseignés pour les années 1994 à 2000, nous observons également des corrélations faibles mais plus importantes en 1995 qu'en $2002(-0,485 \text { contre }-0,479)^{20}$.

2. Les prestations sociales n'ont pas de poids notable dans la détermination des taux moyens de croissance une fois la consommation finale initiale prise en compte. C'est la conclusion que nous tirons de la dizaine de régressions multiples que nous avons testées avec diverses variables explicatives complétant la consommation finale en 1995. Une fois pris en compte l'effet du niveau de vie, le coefficient des prestations n'est pas significatif quelle que soit la date de la série considérée, et l'augmentation associée de la variance expliquée par la prise en compte des prestations sociales (que mesure la variation $\mathrm{du} \mathrm{R}^{2}$ ) est très faible. Il faut donc en conclure qu'elles n'ont pas d'impact mesurable, au niveau de 20 pays, sur les taux moyens de croissance.

3. Les impôts de 2002 contribuent positivement aux taux de croissance une fois la consommation finale initiale et le TSD pris en compte. Cette conclusion correspond au caractère positif du coefficient associé aux impôts dans les régressions multiples lorsque nous prenons en compte un indicateur du niveau de vie en 1995, que ce soit la consommation finale SPA par habitant (notée Cons95) ou le PIB SPA par habitant. De plus, si nous intégrons le TSD en 2002 comme variable supplémentaire, les coefficients des variables TSD (notée Tsd02) et impôt en 2002 (notée Imp02) deviennent significatifs au seuil de $95 \%{ }^{21}$. Nous obtenons alors l'équation de régression suivante pour les taux de croissance $\mathbf{G}$ des 20 pays européens (les écarts-type étant notés entre parenthèses sous les coefficients estimés) :

$$
\mathbf{G}=\underset{(0,49)}{6,33-0,00053 * \text { Cons95 }-7,4 * \text { Tsd02 }+0,00101 * \mathbf{I m p 0 2}}
$$

Cette équation exprime trois régularités, deux que nous avons déjà notées et une troisième moins évidente : d'abord, les pays présentant un retard de leur niveau de vie, comme les PECO ou les pays scandinaves au sortir de la crise de 1994, sont plus poussés à adopter une forte croissance; ensuite, les hauts niveaux de sécurité économique sont associés aux systèmes sociaux arrivés à maturité dans des pays fortement développés dont la pression à la croissance sera donc moins vive ; enfin, le plus haut niveau des impôts est loin de nuire à la croissance et, au contraire, est associé pour une part non négligeable avec des taux de croissance plus élevés, une fois pris en compte le rôle du rattrapage des niveaux de vie dans les pays européens . Mais, à la différence des experts de la Commission européenne, il nous paraît prématuré de déduire tout lien de causalité de telles liaisons entre l'impôt et la croissance.

4. Une croissance notable est loin d'être incompatible avec le maintien de fortes garanties de sécurité. Même si l'équation précédente montre que les hauts niveaux de sécurité

\footnotetext{
${ }^{20}$ Pour les séries de prestations sociales SPA par habitant, si nous prenons la précaution de ne pas prendre en compte les PECO non renseignés (qui ne sont tous disponibles qu'à partir de 2001), nous observons d'abord une faible progression des coefficients de corrélation, depuis -0.484 en 1995 , puis $-0.498,-0.506,-0.510$, puis une lente diminution après 1998 : -0.502, $-0.490,-0,475$, une pause en 2000 à -0.479 , jusqu'à -0.454 pour 2003.

${ }^{21}$ Les probabilités de nullité des coefficients sont respectivement de 0,0001 pour Cons95, de 0,040 pour Imp02 et de 0,052 pour Tsd02, le $\mathrm{F}$ de Fisher valant 19,4 pour une valeur critique de $1,4 * 10^{-5}$ et $l^{\text {'augmentation du } \mathrm{R}^{2}}$ liée à l'ajout des deux variables Imp02 et Tsd02 étant estimée à $+10,6 \%$.
} 
démarchandisée évalués par le TSD ne sont pas associés avec de forts taux de croissance, il reste que, dans le groupe des pays du Nord, quatre sur cinq témoignent de la compatibilité d'une croissance moyenne appréciable, entre $2,9 \%$ et 3,7\%, avec le maintien d'un haut niveau de sécurité démarchandisée (des TSD tous supérieurs à 22\%). Les experts du Forum économique mondial ont abouti à un constat de même nature à partir d'enquêtes auprès des responsables économiques en classant régulièrement trois des pays nordiques parmi les quatre premiers de leur classement mondial de compétitivité. Dans le groupe des pays intermédiaires, cinq sur six maintiennent une croissance au moins égale à 2,2\% alors que leur niveau de sécurité est encore très appréciable (des TSD dépassant 13\%). En définitive, seule l'Italie apparaît à la fois mal placée au niveau de la croissance moyenne $(1,6 \%)$ et du taux de sécurité démarchandisée $(9,3 \%)$.

5. Les prestations sociales ne suffisent pas seules à produire de la sécurité. Le tableau 2 nous permet aussi de constater que les plus importantes sécurités économiques des pays du Nord sont associées pour une grande part avec des niveaux plus élevés de prestations sociales. Le coefficient de corrélation entre le TSD et les prestations sociales SPA par habitant en 2002 est très élevé $(0,859)$, comme d'ailleurs avec le niveau des impôts $(0,839$ en 2002 e 0,859 en 2001). Mais les divergences de classement pour la Norvège, la Finlande ou la Tchéquie montrent qu'il n'y a pas de lien mécanique entre les deux notions. Si les TSD de ces trois pays sont bien mieux classés que leurs niveaux de prestations sociales par tête (respectivement aux rangs 1,5 et 9 contre 10,11 et 14), c'est parce que leurs politiques de lutte contre les inégalités y tiennent une grande priorité. Leurs taux de pauvreté s'en trouvent fortement comprimés et leurs taux d'insécurité économique également. Ce résultat témoigne de l'importance de la composante politique qui peut conférer des significations très différentes à des efforts équivalents en matière de prestations sociales. De ce point de vue, les bons résultats du groupe des pays du Nord correspondent pour une bonne part à l'orientation universaliste de leurs politiques sociales, lesquelles se traduisent par exemple aussi bien dans des mesures de lutte contre l'exclusion, dans des programmes de résorption des discriminations, ou dans des combats prolongés pour l'égalité entre femmes et hommes.

\section{Conclusion}

La comparaison des contributions des prestations sociales à la sécurité démarchandisée que nous avons effectuée dans vingt pays européens nous conduit à deux conclusions claires et à une réponse provisoire qui mériterait d'être approfondie. D'abord, la diversité des résultats obtenus en matière de sécurité économique par les différents pays est considérable. Le taux de sécurité démarchandisée (TSD) de la Norvège est en effet près de dix fois plus important que celui de la Lituanie $(27,9 \%$ contre $2,9 \%)$. Mais il est vrai que ce taux représente une différence, entre les ressources indépendantes du marché correspondant aux prestations sociales et l'insécurité économique associée à la population vivant en-dessous du seuil de pauvreté. Effectuant la synthèse entre deux aspects de la sécurité, ce taux est de ce fait plus sensible à l'effet de ciseau associé d'un côté à la dimension des ressources des politiques sociales et, de l'autre côté, à leurs résultats en matière de pauvreté. Cette diversité des résultats en matière de TSD est bien plus importante que celle des prestations sociales, ce qui souligne en quoi la sécurité économique démarchandisée exprime aussi pour une large part les conséquences des politiques sociales concernant la pauvreté, les discrimination et, plus généralement, les inégalités.

Un deuxième ensemble de conclusions correspond aux caractéristiques des quatre groupes assez typés de pays européens que nous avons constitués du point de vue de la sécurité économique démarchandisée. Le groupe des cinq pays du Nord, à la fois plus productifs, 
ayant un niveau de vie plus élevé et assurant une plus grande sécurité économique à leur population, témoigne en particulier de la compatibilité des objectifs des politiques sociales avec les contraintes du maintien d'une haute compétitivité. Dans le groupe des pays intermédiaires, les systèmes de prestations sociales sont en grande partie arrivés à maturité. Mais leurs insuffisances permettent d'observer en quoi la configuration politique de certains pays les rend moins performant en matière de sécurité économique, même si par exemple les prestations sociales par habitant en France sont au deuxième rang des 20 pays. Le groupe des pays du Sud rassemble des pays dont les systèmes de prestations sociales présentent de grandes insuffisances, soit qu'ils sont en voie d'édification rapide comme la Grèce ou le Portugal, soit qu'ils sont handicapés par des restes de corporatisme comme en Italie ou en Espagne. A l'autre extrême, le groupe des pays d'Europe centrale et orientale témoigne de retards importants en matière de reconstitution et d'édification d'un ensemble de prestations sociales. Les insuffisances de leur niveau de vie se manifestent inversement dans la vigoureuse impulsion de leur processus de croissance.

Quant à la question de la contribution des prestations sociales à la croissance que nous posions en introduction, si notre détour par la sécurité économique ne l'a pas résolue, il a au moins permis de la faire avancer sérieusement. Les corrélations et régressions que nous avons rassemblées ont permis non seulement d'infirmer mais aussi de renverser le sens de l'hypothèse de l'influence décisive du poids de la fiscalité : si les prestations sociales n'ont pas d'influence mesurable sur le taux de croissance, il semble bien que le niveau des impôts y soit associé positivement. L'optimum de taxation ne semble en effet avoir de portée que pour des économies théoriques abstraites et éloignées des formations sociales complexes que constituent les pays européens. Même si les contraintes du financement des prestations sociales jouent un rôle que nous ne pouvons exclure, nos corrélations montrent que leur association avec les taux moyens de croissance est bien plus complexe ; leur contribution est difficile à caractériser du fait qu'elle se combine avec les conséquences d'autres effets massifs sur la croissance tel que celui du rattrapage des niveaux de vie. Il reste que des recherches complémentaires seraient fort utiles concernant les contributions de la sécurité économique, et donc des prestations sociales, à la compétitivité et à la croissance, en particulier pour expliquer les succès des pays du Nord en la matière.

\section{Sources bibliographiques}

ANDRÉ C. (1995), «État-providence et compromis institutionnalisés. Des origines à la crise contemporaine », in BOYER R. et SAILLARD Y. (éd. 1995), Théorie de la régulation.

L'état des savoirs, Paris : La Découverte, p 144-161.

BOYER R. (2001), Régulation, régulations, Paris : La Découverte.

CASTEL R. (2003), L'insécurité sociale. Qu'est-ce qu'être protégé ?, Paris : Seuil.

COMMISSION EUROPÉENNE, Emploi, Affaires sociales et Égalité des chances (2004)

Rapports nationaux sur les stratégies de la protection sociale et de l'inclusion sociale 20062008, Bruxelles, Commission européenne.

CORNILLEAU G. (2006), «La protection sociale et ses difficultés de financement », in Le modèle social français, Cahiers français, $\mathrm{n}^{\circ} 330$, Paris :La Documentation française

DELORME R. ; ANDRÉ C. (1983), L'État et l'économie, Paris : Seuil.

ESPING-ANDERSEN G. (1990), The Three Worlds of Welfare Capitalism, Cambridge:

Polity Press, Trad. : Les trois mondes de l'État-providence, Paris: PUF, 1996.

EUROSTAT (1999), Dépenses et recettes de protection sociale 1980-1997, Luxembourg, Eurostat.

GADREY J. (2002), «De la croissance au développement : quels indicateurs alternatifs ?», 
dans Futuribles, p 39-71.

MENAHEM G. (2007), «The Decommodified Security Ratio: A Tool for Assessing European Social Protection Systems", International Social Security Review, Geneva, n 4.

MENAHEM G. ; CHERILOVA V., «Inégalités de sécurité économique et aide à la famille dans l'Union européenne», Recherches et Prévisions, n79, 2005, pp. 83-95.

OSBERG L.; SHARPE A. (2002), « An Index of Economic Well-being for Selected OECD Countries », Review of Income and Wealth, Groningen, Netherlands, p 291-316.

PETRASOVA A. (2007), «Social protection in the European Union », Statistics in focus, $\mathrm{n}^{\circ}$ 99, Luxembourg, Eurostat.

POLANYI K. (1944), The Great Transformation, Boston, USA: Beacon Press.

RHODES M. ; PALIER B. (1997), «Conclusion générale», dans «Rencontres de Florence Comparer les systèmes de protection sociale en Europe du Sud», Revue française des affaires sociales, $57^{\text {ème }}$ année, 4(numéro spécial), p 607-612.

SAEZ E. (2004), «Reported Incomes and Marginal Tax Rates, 1960-2000 : Evidence and Policy Implications », NBER, document de travail $n^{\circ} 1027$.

STROBEL P. (2003), «Présentation : le modèle nordique de protection sociale sous le choc des réformes», dans «L'État providence nordique», Revue française des affaires sociales, $57^{\text {ème }}$ année, 4(numéro spécial), p 7-16.

WORLD ECONOMIC FORUM (2005), The Global Competitiveness Report 2005-2006, in GADREY J., En finir avec les inégalités, Paris : Mango éditions, p 147-148.

Remerciements : Je remercie le referee anonyme et le professeur Jean Gadrey pour le soin de leur relecture et la qualité des remarques qu'ils ont formulées, lesquelles m'ont incité à apporter de considérables améliorations à mon projet d'article initial. Je suis bien entendu seul responsable des erreurs et insuffisances qui pourraient subsister.

\section{ANNEXES}

\section{Une source : Eurostat}

Eurostat et, en particulier, sa base NewCronos ont permis de rassembler des statistiques des populations, des différents revenus et prestations sociales et des taux de pauvreté pour un grand nombre de pays européens. À la date des interrogations, les statistiques de revenu disponible et de seuil de pauvreté n'étant accessibles ni pour deux pays de l'Europe des 15, le Luxembourg et l'Irlande, ni pour quatre arrivants, Chypre, la Hongrie, Malte et la Slovénie, il a été nécessaire de se limiter à 19 des 25 pays de l'Union européenne en 2002. En revanche, nous avons pu y rajouter la Norvège, mais seulement pour 2001 et 2002.

Comme on peut le visualiser sur le graphique 2, ces statistiques n'ont pu être rassemblées pour les neuf années 1995-2003 que pour dix pays membres de l'Europe des 15, les données de la Finlande n'étant disponibles qu'à partir de 1996, celles de la Suède qu'à partir de 1997 et celles de l'Italie n'étant pas disponibles pour 2003 à la date de nos investigations. L'évolution du TSD est ainsi retracée sur huit années pour onze pays (de 1996 à 2003), et sur six années pour treize. La vague des intégrations dans l'UE à partir de 2000 s'est traduite par un élargissement de la base de Eurostat, ce qui permet de calculer le TSD pour 17 pays de 2000 à 2002 et pour 19 en 2001 et 2002. Au total, seule l'année 2002 permet de réunir les données nécessaires au calcul du TSD pour 20 pays européens, dont un non communautaire, la Norvège. 
On a utilisé la nomenclature SESPROS ${ }^{22}$ des prestations sociales en huit postes établie et harmonisée pour la base NewCronos. Quelles que soient les limites de cette nomenclature, elle a l'avantage de permettre de comparer des systèmes très différents, tant par la nature des droits sociaux qui les fondent que par le contenu des prestations qu'ils génèrent ou par l'orientation des politiques qu'ils soutiennent.

\section{Les étapes du calcul du TSD}

Le taux de sécurité démarchandisée ou TSD, est un ratio qui met en relation trois termes : il évalue un numérateur, la somme des ressources de sécurité démarchandisée, relativement à un dénominateur, le revenu disponible moyen des actifs en emploi, et il en soustrait une évaluation de l'insécurité économique. Nous pouvons décomposer son calcul en trois étapes. Dans chacune d'elles, il a été nécessaire de définir plusieurs conventions de calcul afin de dépasser les obstacles liés à la difficulté à décomposer les données de Eurostat selon les définitions particulières du TSD.

\section{Le calcul des ressources démarchandisées}

L'ensemble des prestations sociales définies dans la base NewCronos a été considéré comme relevant de décisions pour une large part non marchandes. En conséquence, voulant évaluer les ressources de sécurité démarchandisée pour les individus, nous avons considéré que le numérateur est égal à la somme moyenne par adulte des prestations sociales recensées par Eurostat déduction faite d'une estimation des impôts sur les prestations imposables.

En l'absence de décomposition selon le caractère imposable des huit catégories de prestations, nous les avons réparties en deux groupes. Ont été considérées comme en moyenne imposables les quatre catégories suivantes : les retraites, les pensions de réversion et les allocations de chômage (même si nombreux sont les allocataires de tels revenus qui ne sont pas imposables). Ont été considérées comme en moyenne non imposables les indemnités maladie, invalidité, les allocations de soutien aux familles, d'aide au logement et de lutte contre l'exclusion (même si plusieurs de ces revenus se trouvent être pris en compte dans les ménages imposables). Nous avons appliqué aux prestations dites imposables un taux moyen d'imposition estimé comme le rapport entre le total des impôts sur les personnes (revenus et patrimoines) et le total des revenus imposables (de l'activité, des prestations et du patrimoine)

\section{L'évaluation du dénominateur}

Le taux de ressources démarchandisées évalue les prestations sociales moyennes par adulte relativement à un indicateur du niveau de vie moyen. Il est donc égal au rapport entre le numérateur précédemment calculé et un dénominateur estimant le revenu disponible moyen des actifs ayant un emploi. Ce dernier revenu est égal à la somme moyenne des rémunérations de l'activité professionnelle (indépendante et salariée) nettes des cotisations sociales, d'une estimation des revenus de la propriété touchés par les adultes en emploi, d'une estimation des prestations sociales versées aux personnes en emploi, déduction faite d'une estimation de l'impôt sur les revenus des personnes physiques (pour l'activité et pour le patrimoine).

Pour estimer les différents revenus des personnes en emploi, nous avons supposé qu'ils sont en moyenne équivalents à ceux des personnes sans emploi (du fait de la retraite ou du chômage), ce qui permet de répartir proportionnellement aux populations concernées les revenus de la propriété et les prestations sociales universelles. Le taux moyen d'imposition précédemment évoqué a été appliqué à l'estimation correspondante des revenus imposables pour déterminer une estimation de l'impôt venant en déduction du revenu disponible.

\footnotetext{
${ }^{22}$ SESPROS est le sigle français pour désigner la nomenclature plus connue pour son appellation en anglais EESPROS : «European System of Integrated Social Protection Statistics ».
} 


\section{Le calcul de l'insécurité économique}

Dans un pays donné, la population considérée comme pauvre est supposée par définition comme ne disposant pas de sécurité économique : le taux de sécurité démarchandisée de cette population doit donc être fortement pénalisé quand bien même elle dispose de faibles ressources. Par référence à la convention adoptée par Eurostat définissant le seuil de pauvreté pour un ménage comme le revenu par unité de consommation égal à $60 \%$ du revenu médian d'une population, nous avons considéré que la pénalisation correspondant au fait de se trouver dans une situation de pauvreté est égale à $60 \%$ du revenu de référence dans cette population.

L'insécurité économique d'une population vient donc en déduction de son taux de ressources démarchandisées. Cette pénalisation concerne la part de la population du pays considérée comme pauvre, soit selon Eurostat vivant dans des ménages dont le revenu par unité de consommation n'atteint pas le seuil de pauvreté. Elle est égale à 0,6 fois la part de la population pauvre dans la population adulte.

En conclusion, il est utile de rappeler la nécessité d'établir de telles conventions de calculs, même si nous sommes conscients de leur caractère approximatif et en partie arbitraire. Mais il est possible de les améliorer. C'est une raison pour les publier afin de les soumettre à la critique, en particulier dans l'espoir de susciter des suggestions de la part des lecteurs. 